\title{
Systematic review of economic evaluations of exercise and physiotherapy for patients treated for breast cancer
}

\author{
Kamran Ahmad Khan ${ }^{1} \oplus \cdot$ Bruno Mazuquin $^{1} \cdot$ Alastair Canaway $^{1} \cdot$ Stavros Petrou $^{1} \cdot$ Julie Bruce $^{1}$
}

Received: 25 February 2019 / Accepted: 10 April 2019 / Published online: 17 April 2019

(c) The Author(s) 2019

\begin{abstract}
Purpose Treatments for breast cancer can lead to chronic musculoskeletal problems. This study aimed to systematically review the evidence surrounding the cost-effectiveness of exercise and physiotherapy interventions aimed at reducing the risk of physical symptoms and functional limitations due to breast cancer treatment.

Methods A systematic review of the cost-effectiveness of exercise and physiotherapy interventions during and following treatment for breast cancer was undertaken according to PRISMA guidelines. Literature searches were carried out in Ovid MEDLINE, Ovid Embase, Web of Science, EconLit, CINAHL, PsycINFO, Scopus and the Cochrane Library. Costeffectiveness evidence was summarised in a descriptive manner and studies were assessed using quality appraisal tools. The review protocol was registered on PROSPERO.

Results A total of 7783 articles were identified and seven were included in the final review. Five studies undertook trialbased economic evaluations, whereas two studies conducted economic evaluation based on decision models. One study was a cost-effectiveness analysis (CEA), three undertook stand-alone cost-utility analyses (CUA) and three studies were combined CEAs and CUAs. Three studies reported favourable cost-effectiveness results for different exercise or physiotherapy interventions. In contrast, four studies found that exercise and physiotherapy interventions were not cost-effective on the basis of quality-adjusted life year outcomes.

Conclusions The evidence surrounding the cost-effectiveness of exercise and physiotherapy interventions for the treatment of breast cancer remains sparse with contrasting conclusions. Future research should particularly aim to broaden the evidence base by disentangling the contributing effects of frequency, intensity, time and type of exercise and physiotherapy interventions on cost-effectiveness outcomes.
\end{abstract}

Keywords Exercise $\cdot$ Rehabilitation $\cdot$ Cost-effectiveness $\cdot$ Systematic review $\cdot$ Economic evaluation

\section{Introduction}

Breast cancer is the most common form of cancer amongst women in the United Kingdom (UK) with approximately 50,000 new cases diagnosed each year [1]. Most women diagnosed with breast cancer have surgery to the breast and axilla, with many also requiring radiotherapy and

Electronic supplementary material The online version of this article (https://doi.org/10.1007/s10549-019-05235-7) contains supplementary material, which is available to authorized users.

Kamran Ahmad Khan

k.a.khan@warwick.ac.uk

1 Warwick Clinical Trials Unit, Warwick Medical School, University of Warwick, Gibbet Hill Road, Coventry CV4 7AL, UK chemotherapy $[2,3]$. These treatments can affect the muscles, nerves and lymphatic vessels in the shoulder and upper body, resulting in musculoskeletal problems such as limited range of motion, weakness, persistent pain, altered sensations and lymphoedema $[4,5]$. Studies suggest that between 10 and $64 \%$ of women have symptoms in their arm or shoulder up to 3 years after treatment [6]. These persistent symptoms can delay recovery, limit daily activities and impair health-related quality of life (HRQoL). It is important that the UK National Health Service (NHS) and health systems in other countries provide adequate care for women to ensure recovery and return to usual activities after cancer treatment.

Exercise interventions may alleviate the side-effects of cancer treatment with several systematic reviews of literature suggesting they may be clinically effective [7-10]. McNeely et al. [8]., for example, reported that exercise improves 
HRQoL and physical capacity and reduces fatigue in breast cancer patients. Furthermore, physical activity can improve cardiorespiratory capacity and well-being in cancer patients [9].

Although exercise interventions have been shown to be clinically effective in several studies, information on their cost-effectiveness is sparse. Two systematic reviews, one investigating the cost-effectiveness of exercise-based interventions in the treatment of various chronic diseases [11] and the other the cost-effectiveness of cancer rehabilitation [10], identified only two economic evaluations of physical activity interventions for breast cancer patients $[12,13]$. The first study reported that a home-based self-managed physiotherapy intervention and a supervised group-based exercise intervention with psychosocial support were more cost-effective than usual care [12]. In contrast, the second study concluded that a home-based self-managed exercise intervention was not cost-effective compared to an active control consisting of flexibility and relaxation activities after breast cancer surgery [13].

Given the limited resources in public health systems, healthcare interventions should seek to maximise health benefits or broader measures of social welfare with the resources available. To achieve efficient resource allocation, only methods of proven cost-effectiveness should be adopted for routine use in the NHS and other publicly funded health systems [14-17]. Therefore, the aim of this study was to systematically review evidence on the cost-effectiveness of exercise and physiotherapy interventions for the treatment of breast cancer to inform policy decisions in this clinical area.

\section{Methods}

A systematic review of the literature, following Preferred Reporting Items for Systematic Review and Meta-Analyses (PRISMA) guidelines [18], on the cost-effectiveness of exercise and physiotherapy during and following treatment for breast cancer was undertaken. Literature searches were carried out in Ovid MEDLINE, PubMed, Ovid Embase, Web of Science, EconLit, CINAHL, PsycINFO, Scopus and The Cochrane Library (including the NHS Economic Evaluation Database (NHSEED) electronic databases with time horizons covering inception of the databases to 24 September 2018. Biomedical databases were searched using various combinations of keywords and medical subject headings (MeSH) based on terms relevant to breast cancer, physiotherapy, exercise or physical activity and economic evaluation. Further details on the search strategies applied to each database are available in the Online Resource 1. Searches were not limited by date of publication or language. The review protocol was registered on PROSPERO (CRD42018108978).

\section{Selection criteria}

Economic evaluations of exercise and physiotherapy interventions for breast cancer patients were considered. Eligible types of economic evaluations included cost-effectiveness analyses, cost-benefit analyses, cost-utility analyses, cost consequences analyses and cost-minimisation analyses. Each study was required to have reported both costs and consequences and compared an experimental intervention to at least one other intervention or control. Participants included in the selected studies were adults with a confirmed breast cancer diagnosis who were undergoing or had received treatment, including any surgical removal of breast tumour, e.g. mastectomy (simple, modified or radical), local wide excision or lumpectomy and/or axillary surgery (lymph node dissection/clearance or sentinel lymph node biopsy (SNB/SNLB) or dissection. An exercise or physiotherapy intervention was defined as one that included an exercise intervention delivered and supported or unsupported by a physiotherapist or other health professional. Comparators included usual care/control, different types of exercises or no exercise. Descriptions of usual care/control were extracted from primary reports. Outcome measures included measures of cost-effectiveness, e.g. an incremental cost-effectiveness ratio (ICER) or a measure of net monetary benefit (NMB). All identified titles and abstracts were screened independently by two authors (KK and BM) and, where relevant, full-text articles were obtained and assessed against the study inclusion criteria. Disagreements at each stage (title and abstract stage, full report stage) were resolved by discussion or referred to a third author (SP) for final assessment.

\section{Data extraction}

Data extraction was carried out by one reviewer (KK) and checked by a second reviewer (BM). Any disagreements were resolved through discussion or through a final assessment by a third reviewer (SP). Data were extracted using a standardised form. Extracted data items included author(s), year of publication, country and setting, patient characteristics, intervention and comparator details, main analytical approaches (e.g. patient-level analysis or decision-analytic modelling) and the primary outcome(s) specified for the economic analysis. In addition, details of estimation and adjustment for HRQoL, key assumptions made in the base case or tested in sensitivity analyses, direct costs (medical and non-medical) and productivity costs estimated, estimates of cost-effectiveness and approaches to quantifying uncertainty (e.g. decision uncertainty to address uncertainty around the value of 
the cost-effectiveness threshold, probabilistic sensitivity analysis to address uncertainty surrounding the value of parameter inputs) were extracted.

\section{Quality assessment}

The quality of reporting by the economic evaluations was assessed using the Consolidated Health Economic Evaluation Reporting Standards (CHEERS) checklist [19]. The quality of each economic evaluation was scored using CHEERS criteria, which allows overall scores from 0 to 24 (24 representing the best score possible). In addition, the methodological quality of any randomised controlled trial underpinning an economic evaluation was assessed using the Cochrane Collaboration's risk of bias tool [20]. The risk of bias domains included random sequence generation, allocation concealment, blinding of participants, blinding of personnel, blinding of outcome assessment, incomplete outcome data and selective outcome reporting. Each domain was classified as of low, high or unclear risk [20]. Where studies failed to report an item, it was classified as unclear.

\section{Analysis}

Cost data extracted from studies were inflated, where necessary, to 2016 prices using the relevant country-specific Gross Domestic Product (GDP) deflator index, and subsequently converted, where necessary, from their respective currencies into US dollars using purchasing power parities supplied by the Organisation for Economic Co-operation and Development (OECD) [21]. For studies that failed to report their currency price dates, it was assumed that the costs used in the valuation process applied to the financial year prior to the publication of the study.

Methodological variations between studies, including variations in underpinning health care practices across jurisdictions and variations in the relative prices of labour and capital inputs across jurisdictions, prevented a pooling of economic data akin to meta-analyses performed on clinical effectiveness estimates. Rather, cost-effectiveness estimates and broader economic outcomes are presented in a descriptive manner according to broad economic design.

\section{Results}

\section{Search results}

In total, 14,636 records were identified from the bibliographic searches. After removing 6853 duplicates, 7783 titles and abstracts were reviewed and 7773 articles were subsequently excluded at the title and abstract screening stage (Fig. 1). Common reasons for exclusion were that the studies were not economic evaluations, the population did not include breast cancer patients or the intervention was not exercise or physiotherapy based. Ten articles fulfilled screening criteria and were retrieved for full-text analysis; of these, seven fulfilled the study inclusion criteria. Two studies were excluded at the full report stage because they were not economic evaluations [22, 23], whilst a third study by Kampschoff and colleagues [24] presented only aggregated results across breast and colon cancer patients and it was not possible to obtain data only for breast cancer (Fig. 1). Of the seven included studies, one study by Perrier and colleagues [25] was reported as a conference poster and further details were obtained directly from the authors.

\section{Study characteristics}

Descriptive information (study design, patient characteristics, interventions, outcomes) pertaining to each included study is presented in Table 1. Classified by country of origin, three studies were conducted in the Netherlands, three conducted in Australia and one in France. Five studies carried out trial-based economic evaluations [13, 25-28], whereas two studies conducted economic evaluation based on decision models [12, 29]. A decision model theoretically allows for the extrapolation of costs and effects beyond the time horizon of trial data, can reflect all appropriate evidence, can compare all relevant options and can make head-to-head comparisons of alternative competing interventions when relevant trials do not exist [30,31]. The study by Gordon et al. [12] made head-to-head comparisons of alternative competing interventions without trial-based data, whereas Mewes et al. [29] used decision modelling to extrapolate costs and effects beyond the time horizon of the trial data used [32].

\section{Interventions and outcomes}

The type of physiotherapy and exercise interventions evaluated by the studies included home-based self-managed exercises [13, 28, 29], home-based self-managed and supervised physiotherapy [12], home-based supervised exercises with different delivery methods (face-to-face or over-thetelephone) [26] and group-based supervised exercise programmes $[12,25,27,28]$. The physiotherapy and exercise interventions targeted a range of health and fitness goals including strength and flexibility, balance, endurance and overall fitness (Table 1). The range of control interventions included usual care [12, 26, 27, 29], a sham intervention (active control of flexibility and relaxation activities) [13], dietary advice [25] and usual care with no routine exercise [28]. For the five studies using trial data, the primary outcome measures included the self-report Functional Assessment of Cancer Therapy-Breast Cancer version 4 


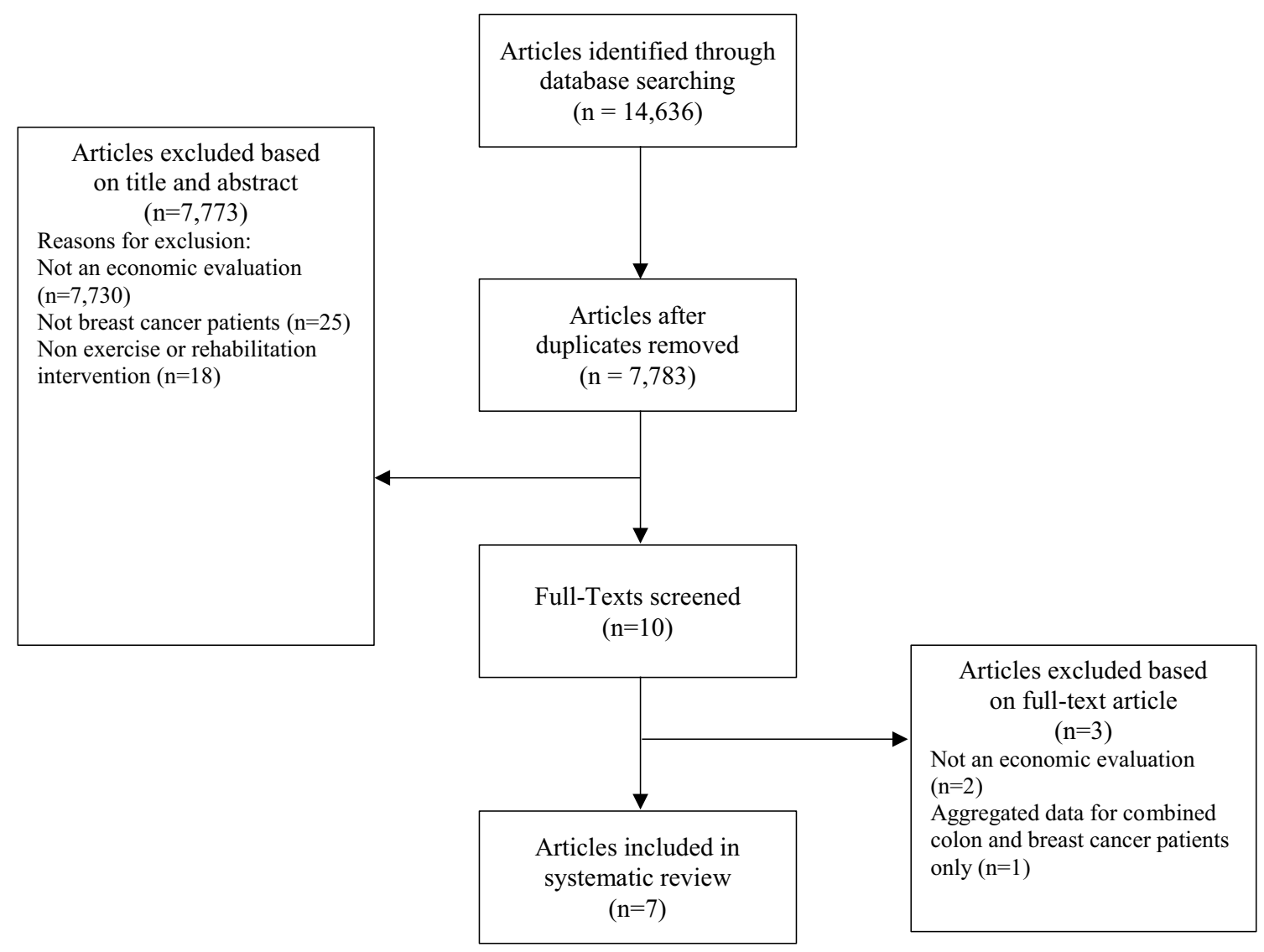

Fig. 1 PRISMA flow diagram

(FACT-B + 4) [26], EuroQol 5-dimension questionnaire 3 level (EQ-5D-3L) [13], European Organisation for Research and Treatment of Cancer core quality of life questionnaire (EORTC QLC-C30) [13], cardiorespiratory fitness [28] and the multidimensional fatigue inventory (MFI) and fatigue quality list (FQI) [27].

\section{Economic evaluations}

Information relating to the characteristics and economic outcomes of the economic evaluations is presented in Table 2. All economic evaluations were published between 2005 and 2018. One study was cost-effectiveness analysis (CEA) [25], three were stand-alone cost-utility analyses (CUAs) $[13,27,29]$ and three were combined CEAs and CUAs [12, 26, 28]. Four studies adopted a societal perspective [12, 13, $27,28]$, whilst one adopted a health care system perspective [29], one a private and service provider perspective [26] and one a national insurance perspective [25]. The mean total costs per patient for delivering group exercise interventions ranged from AUS\$342 (US\$327, 2016 prices) for a home-based physiotherapy intervention [12] to $€ 31,133$ (US\$38,819, 2016 prices) for a home-based, low-intensity, individualised, self-managed physical activity programme with the addition of behavioural reinforcement [28]. The primary measure(s) of health consequence included in the seven economic evaluations fell into the following categories: number of rehabilitated cases and quality-adjusted lifeyears (QALYs) [12, 26]; change in body mass index (BMI) and cardiorespiratory fitness [25]; fatigue and QALYs [28] and QALYs alone [13, 27, 29]. QALYs were derived from the EQ-5D-3L measure in four studies [13, 26-28], whilst in one study [12], QALYs were generated by multiplying period of life by utility scores obtained using a single-item linear analogue scale entitled the Subjective Health Estimation (SHE) scale which had been developed and validated by the International Breast Cancer Study Group [33]. A further study used a mapping algorithm to estimate EQ-5D utility scores from the short-form six dimension health index (SF6D) and then used those values to calculate QALYs [29].

\section{Quality of studies}

The methodological quality assessment of the economic evaluations as judged by the CHEERS checklist produced scores ranging from 19 to 22 (Online Resource 2). For risk 


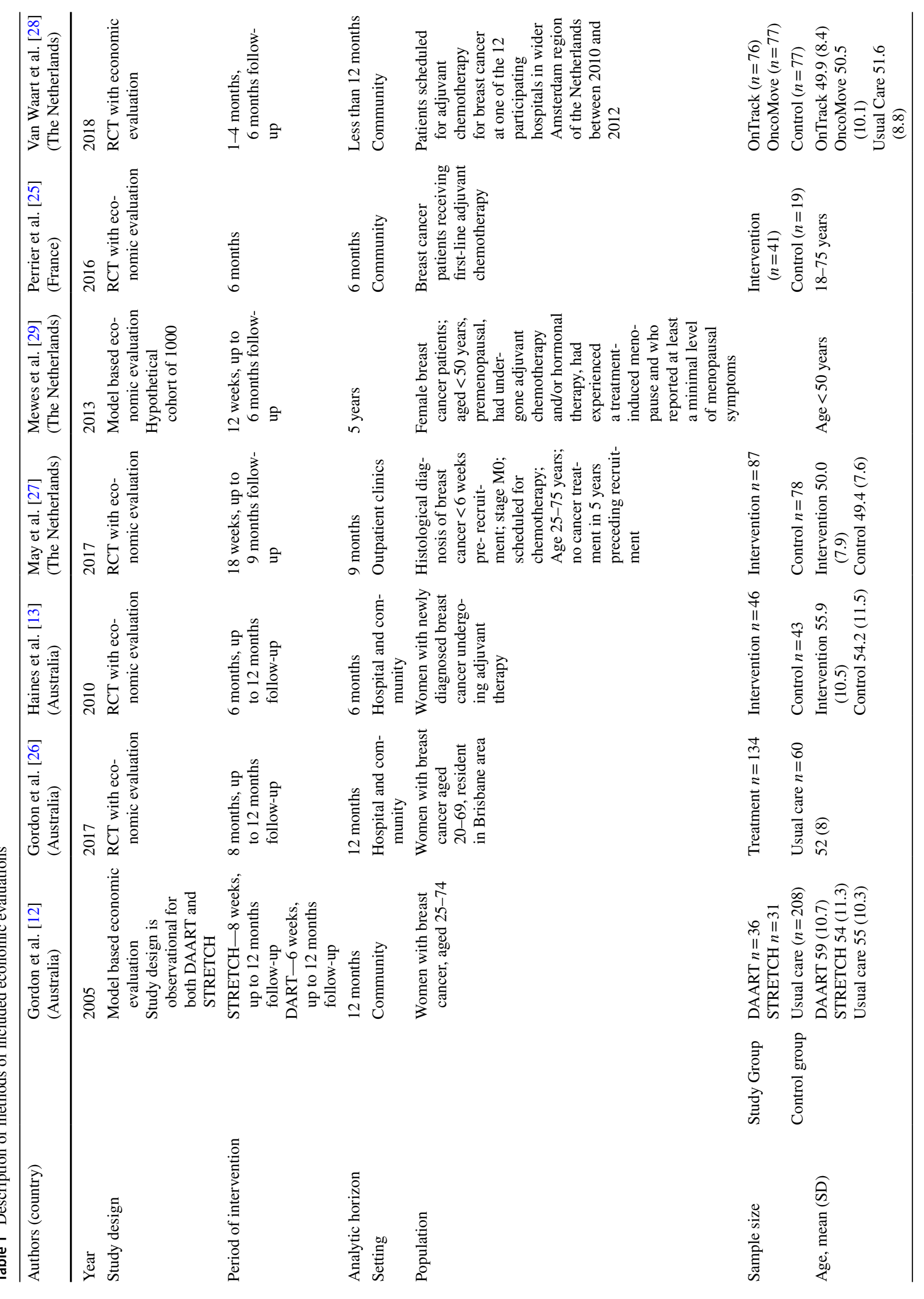




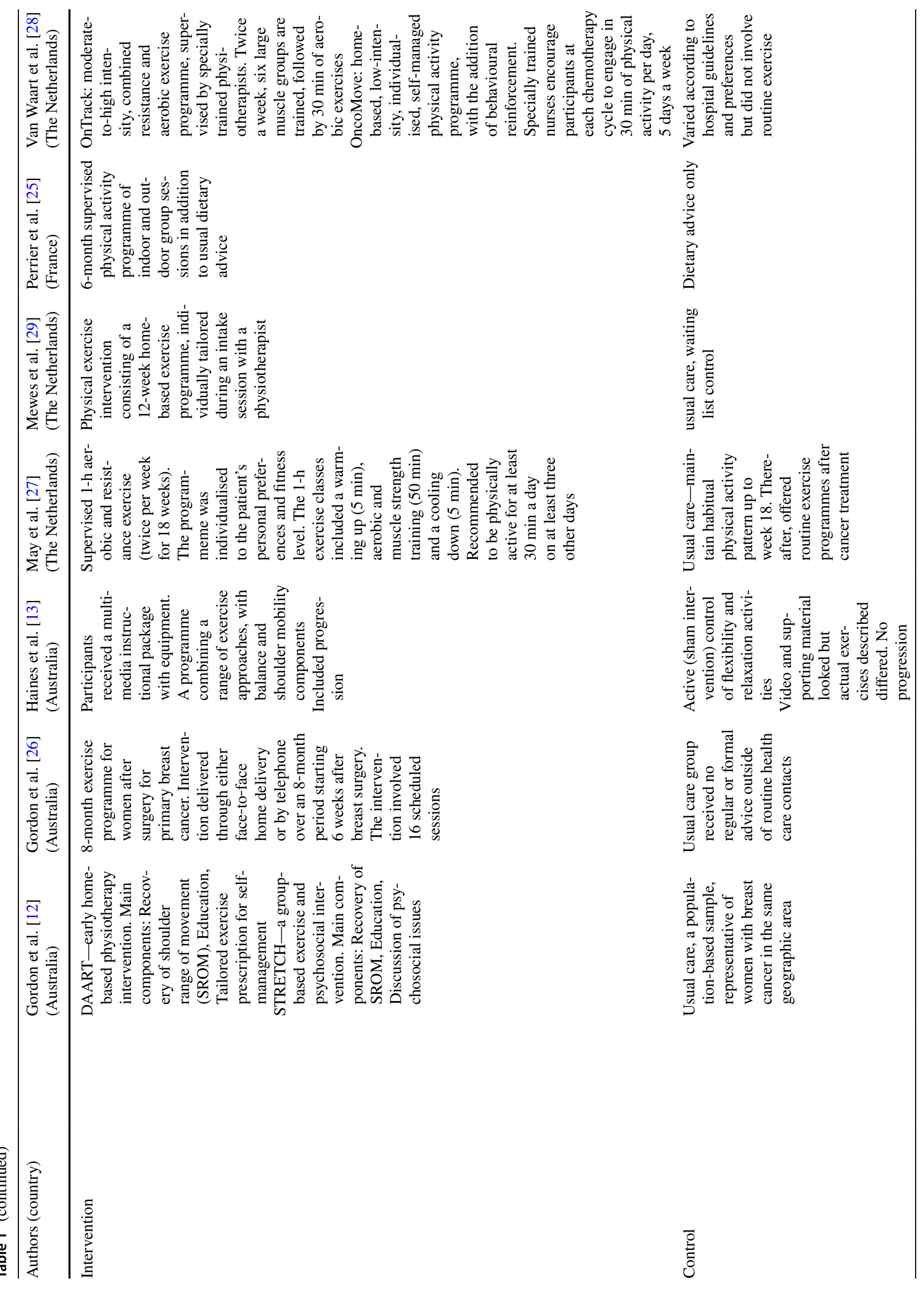


of bias, although there were five studies that carried out trialbased economic evaluations [13, 25-28], Mewes et al. [29] used a decision model that drew upon data from a trial [32]; therefore, risk of bias results are presented for six studies. The methodological assessment of risk of bias was consistent across studies (Fig. 2, Online Resource 3) with the majority of studies considered at low of risk of bias for the majority of domains. However, all studies were considered at high risk of bias for the domain "blinding of participants and personnel" due to the unconcealed nature of the exercise and physiotherapy interventions.

\section{Summary of results of economic evaluations}

The results pertaining to the cost-effectiveness of the exercise and physiotherapy interventions for breast cancer patients evaluated are summarised in Table 2.

\section{Cost-effectiveness studies}

Only one economic evaluation, a study conducted in France, reported non-QALY based results alone and found that a supervised group-based intervention dominated usual care (i.e. generated improved health outcomes and lower overall costs, on average) with a negative ICER of $€-11,159$ per decline in BMI unit [25]. The results for cardiorespiratory fitness also showed that the intervention dominated usual care with a negative ICER of $€-6030$ per estimated aerobic capacity unit gained for $\mathrm{VO}_{2} \max$ [25]. Probabilistic sensitivity analyses conducted by the authors showed that the probability that the intervention is cost-effective reached $56 \%$ based on the BMI outcome and $69 \%$ based on the VO2max outcome [25].

\section{Cost-utility studies}

Three economic evaluations estimated cost-effectiveness results using the QALY framework alone [13, 27, 29]. One Australian study reported that a home-based selfmanaged exercise intervention was not cost-effective, with only a $5 \%$ probability that it was both less costly and more effective than the control [13]. The second study, from the Netherlands, found that a supervised group-based exercise intervention was not cost-effective compared with usual care with an ICER of $€ 291,200$ per QALY gained (US\$375,572, 2016 prices) [27]. Scenario analysis, where the cost-effectiveness was considered from a healthcare perspective, provided similar to results of the baseline analysis [27]. The third study, also from the Netherlands, found that a home-based self-managed exercise intervention generated an ICER of $€ 28,078$ (US\$35,707, 2016 prices) per QALY gained and the authors concluded that this was borderline cost-effective 


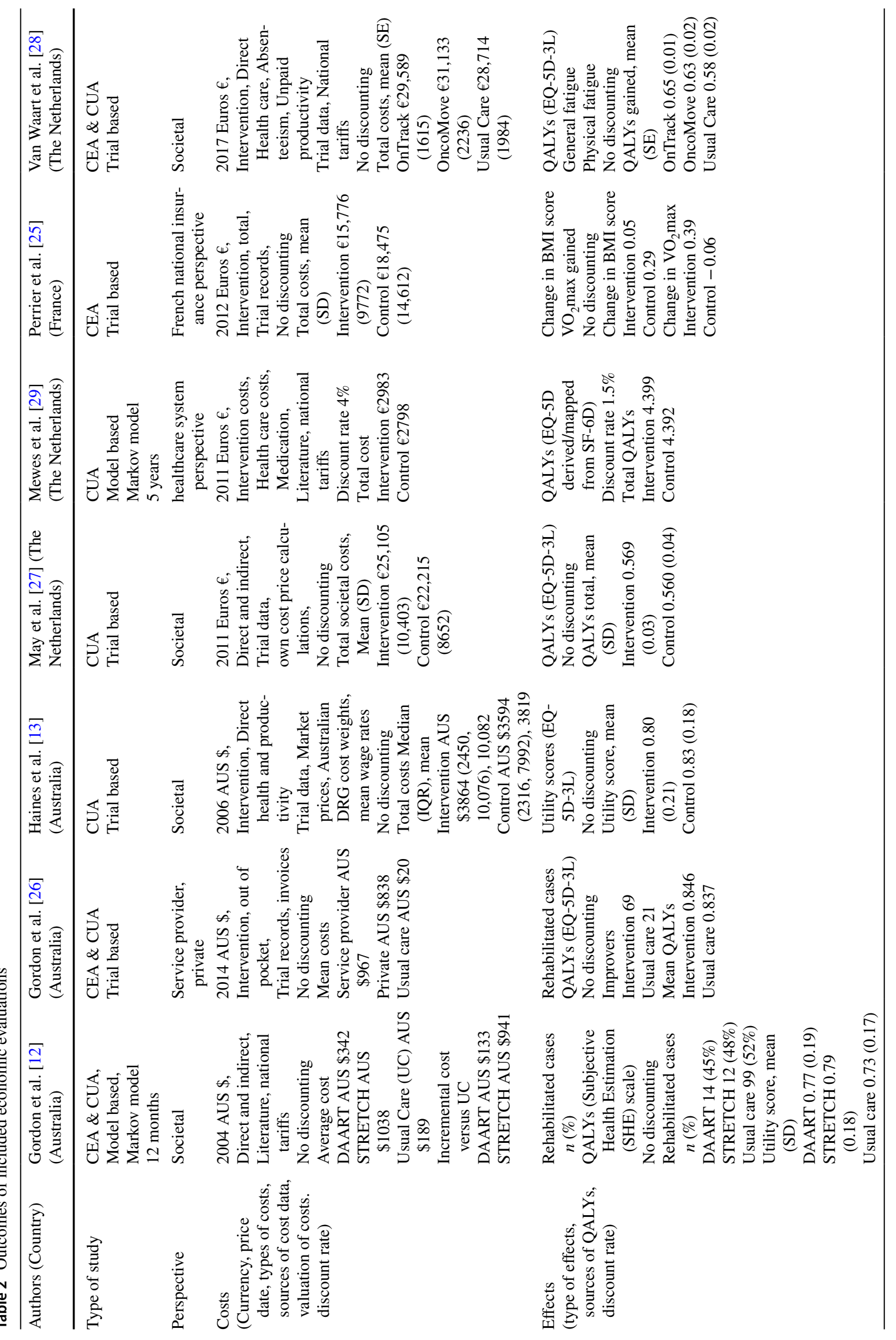




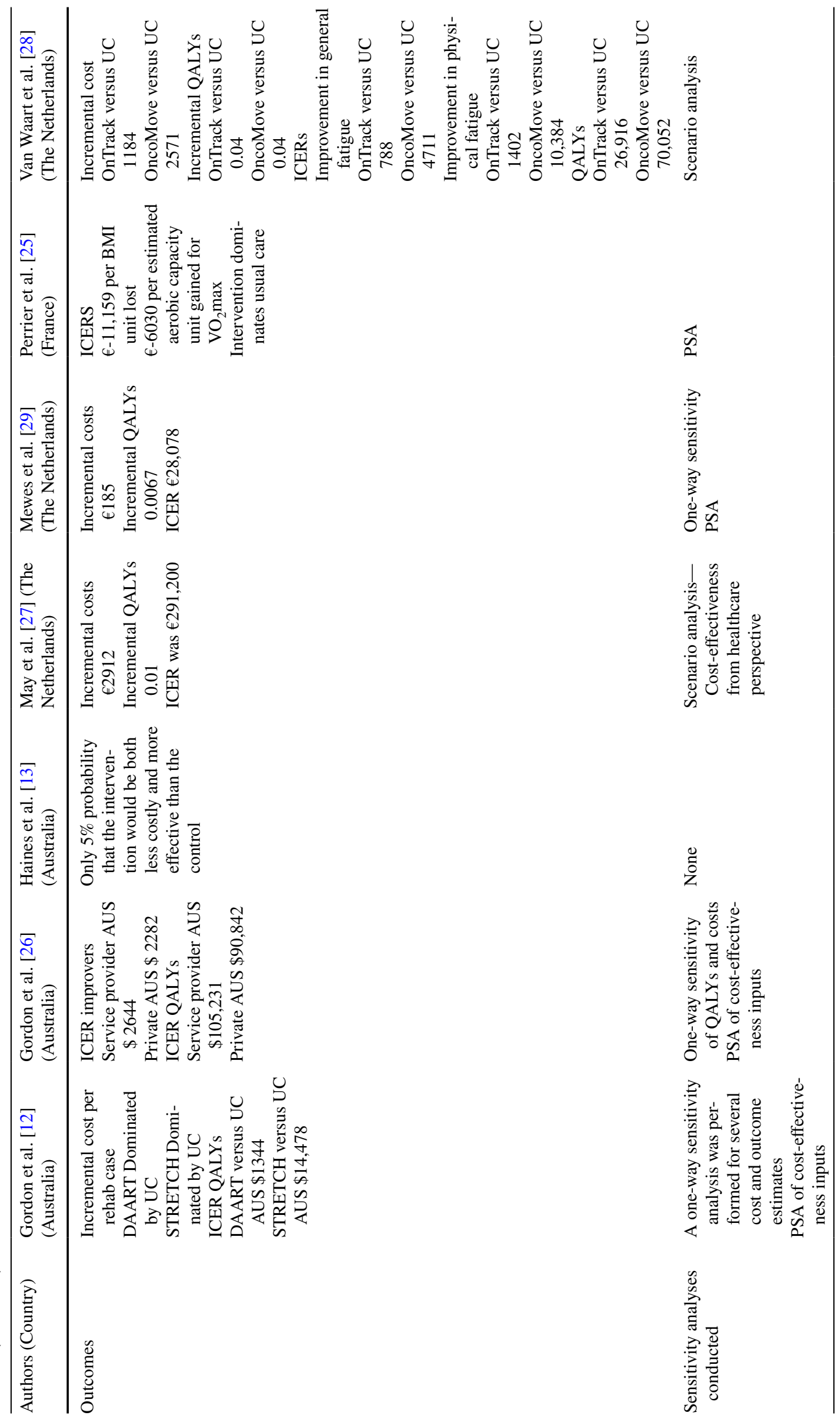




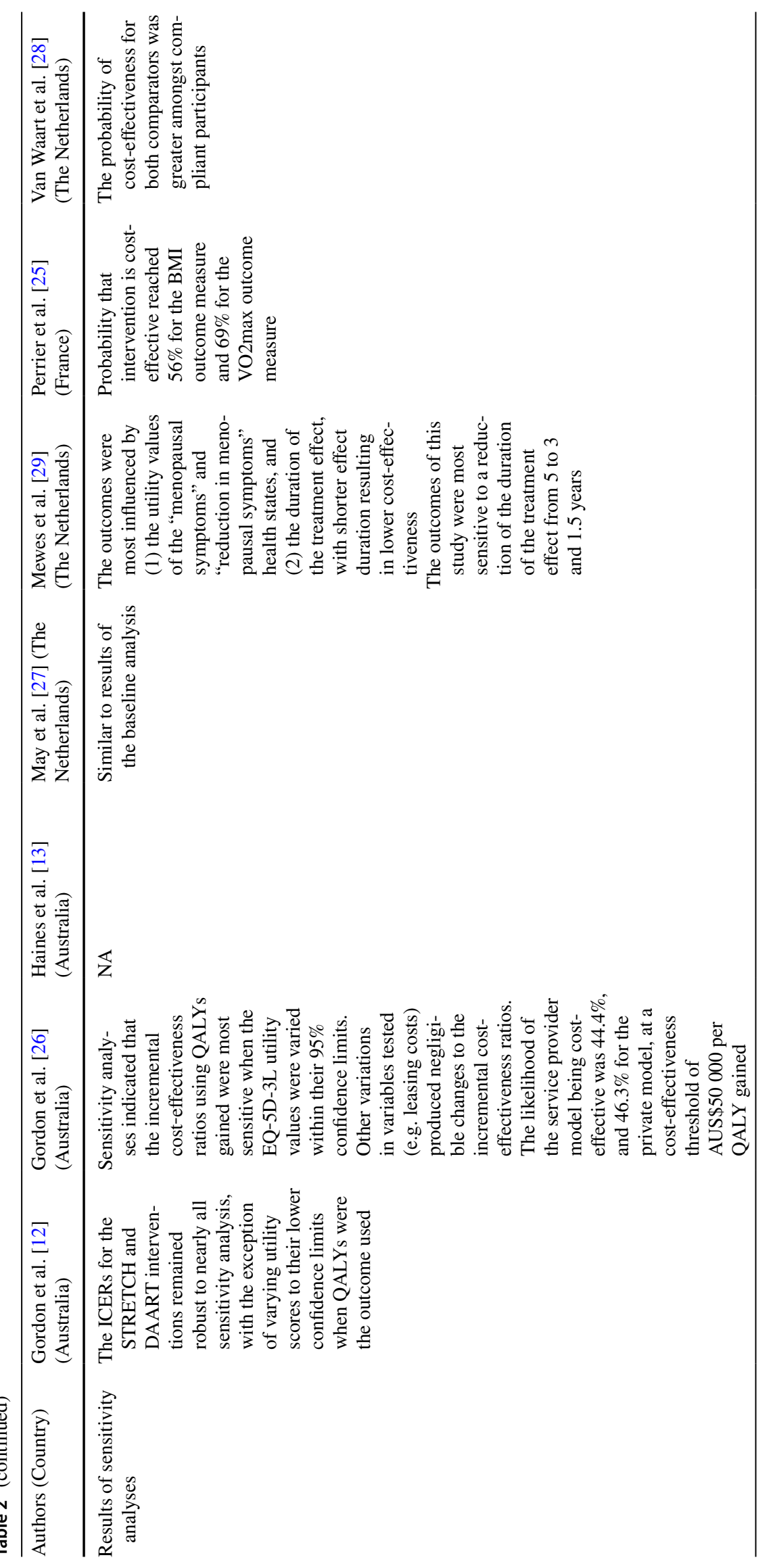




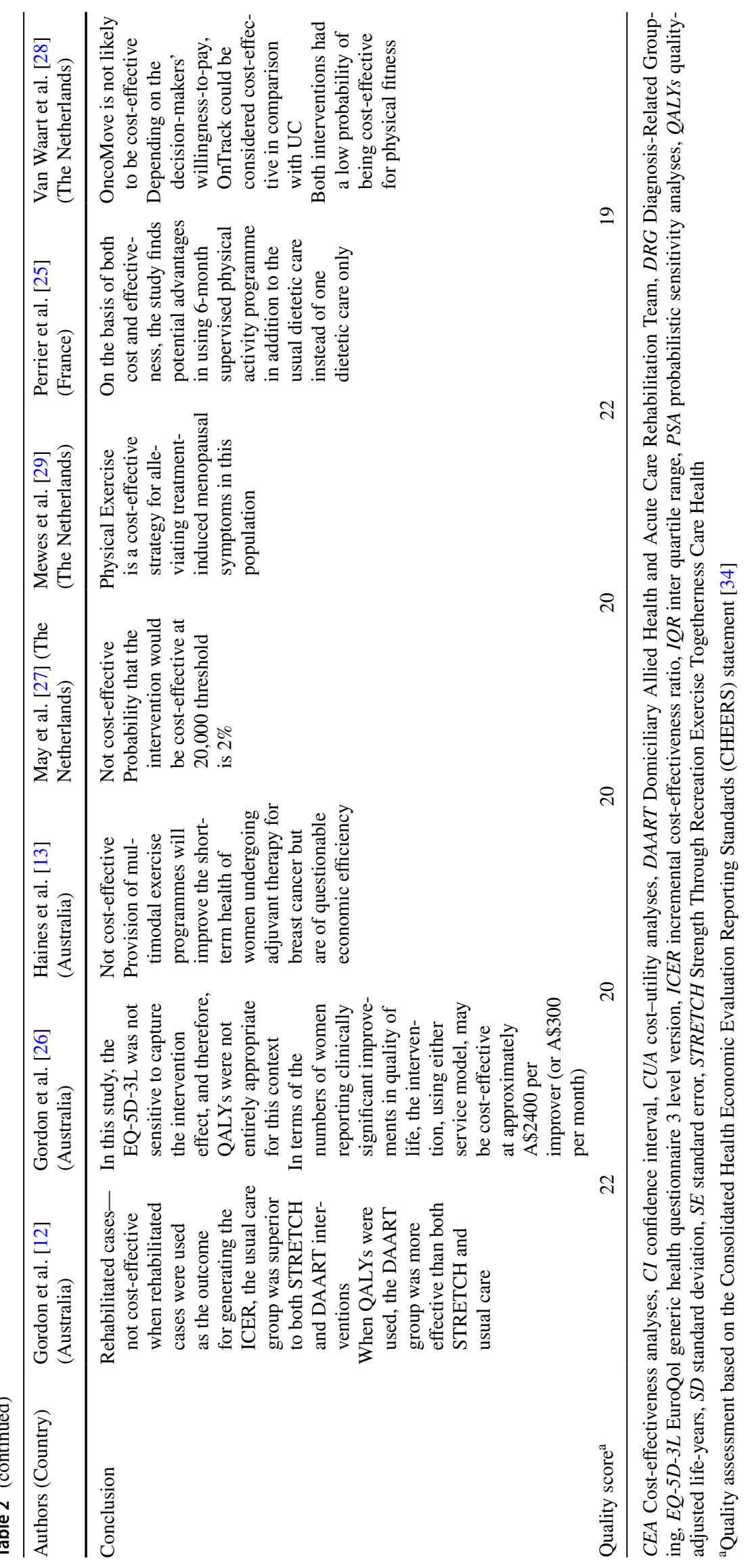


Fig. 2 Risk of bias assessment

a Risk of bias graph

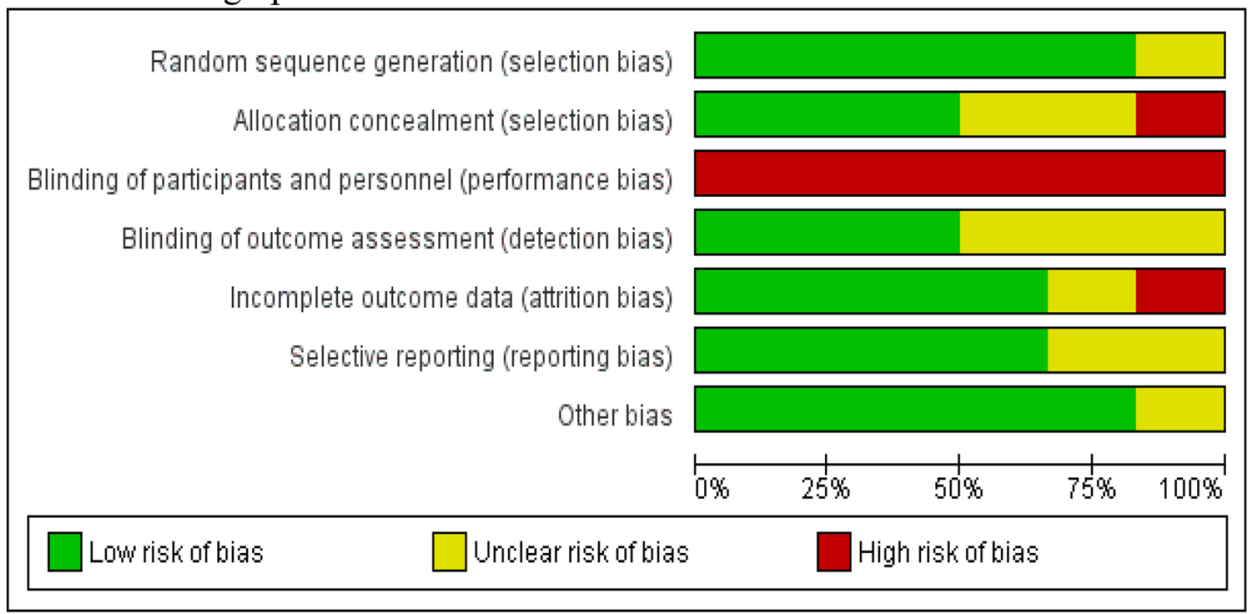

\section{b Risk of bias summary}

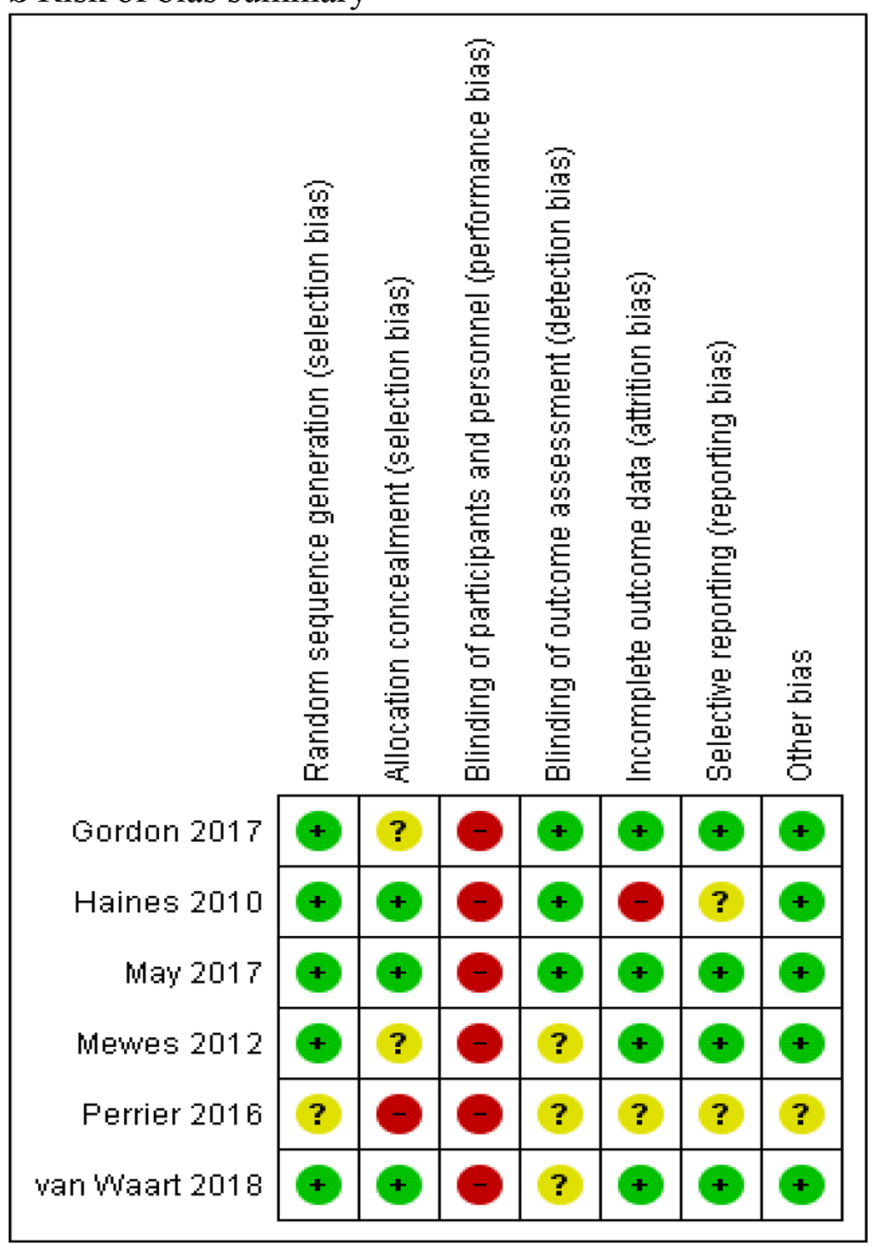

because the ICER of $€ 28,078$ fell below recommended cost-effectiveness thresholds [29]. Sensitivity analyses found that the outcomes were influenced by, first, utility values for the "menopausal symptoms" and "reduction in menopausal symptoms" health states and, second, the duration of the treatment effect, with shorter effect duration resulting in lower cost-effectiveness. The outcomes of this study were most sensitive to a reduction of the duration of the treatment effect from 5 to 3 and 1.5 years [29]. 


\section{Combined cost-effectiveness and cost-utility studies}

Three studies estimated cost-effectiveness results using both QALY and non-QALY frameworks. An Australian study showed that a home-based self-managed and supervised physiotherapy intervention (ICER: AUS\$ 1344 (US\$1284) per QALY, 2016 prices) and a supervised group-based exercise and psychosocial intervention (ICER: AUS\$ 14,478 (US\$13,831) per QALY, 2016 prices) were both more effective than usual care, with the home-based intervention being the more cost-effective of the two experimental interventions [12]. In contrast, the results based on rehabilitated cases showed that usual care was less costly and more effective than both the home-based physiotherapy and group-based exercise and psychosocial interventions [12]. The ICERs for the two experimental interventions remained robust to several sensitivity analyses, with the exception of variations in utility scores to the lower limits of confidence intervals when QALYs were the outcome used. The authors conducted post hoc analyses to check whether self-reported function (FACT$\mathrm{B}+4$ ) (used to estimate rehabilitated cases) and health utility scores (derived from Subjective Health Estimation scale) measured different concepts; they found that the measures were only modestly correlated (coefficient $=0.54, p<0.001$ ), which signified that the two outcome measures were sufficiently different and therefore different cost-effectiveness conclusions were possible given the study design [12].

Another Australian study reported that a home-based supervised exercise intervention was not cost-effective from either a health service provider (ICER: AUS \$ 105,231 (US\$73,786) per QALY, 2016 prices) or a private payer perspective (ICER: AUS\$ 90,842 (US\$63,697) per QALY, 2016 prices) [26]. In contrast, the authors reported that results based on rehabilitated cases showed that the home-based supervised exercise intervention was cost-effective, with an ICER of approximately AUS $\$ 2400$ (US\$1677, 2016 prices) per improver [26]. Sensitivity analyses indicated that the incremental cost-effectiveness ratios using QALYs gained were most sensitive to variations in EQ-5D-3L utility values within $95 \%$ confidence limits. Other variations in variables tested produced negligible changes to the incremental costeffectiveness ratios. The likelihood of the service provider model being cost-effective was $44.4 \%$, and $46.3 \%$ for the private model, at a cost-effectiveness threshold of AUS $\$ 50,000$ per QALY gain. The authors concluded that whilst QALYs are the preferred measure of health consequence in health economic evaluations, there are a couple of reasons why they may not have been appropriate for this clinical context. Firstly, the intervention duration was not expected to extend participant survival during the trial period. Secondly, the mean health utility weight for the study participants $(0.84)$ was similar to that reported for the Australian general population. Therefore, detecting differences in QALYs was deemed unrealistic in their sample [26].

A third study, conducted in the Netherlands, reported that a supervised exercise intervention was borderline costeffective compared to usual care with an ICER of $€ 26,916$ (US\$33,561, 2016 prices) per QALY gained [28]. The authors report that the non-QALY based results for this intervention suggest that it is cost-effective in terms of cost per unit change in general fatigue (ICER of $€ 788$ ), and cost per unit change in physical fatigue (ICER of $€ 1402$ ) [28]. The same study showed that a home-based self-managed exercise with the addition of behavioural reinforcement was not cost-effective compared with usual care with an ICER of $€ 70,052$ (US\$87,347, 2016 prices) per QALY gained [28]. In contrast, the authors reported that home-based selfmanaged exercise with the addition of behavioural reinforcement is cost-effective in terms of cost per unit change in general fatigue (ICER of $€ 4711$ ), and cost per unit change in physical fatigue (ICER of $€ 10,384$ ) [28]. Scenario analyses conducted by the authors found that the probability of cost-effectiveness for both comparators was greater amongst compliant participants [28].

\section{Discussion}

Evidence on the cost-effectiveness of exercise and physiotherapy interventions for the treatment of breast cancer was systematically assessed in this review. We identified only seven studies reporting on the cost-effectiveness of exercise and physiotherapy interventions for breast cancer patients [12, 13, 25-29], which between them evaluated nine different exercise-based interventions. These studies were generally of high quality and at low risk of bias. There have been two previous reviews that have reported evidence on the cost-effectiveness of exercise-based interventions in the treatment of breast cancer. The first review by Roine et al. [11] identified a single study, which reported that a home-based self-managed physiotherapy intervention and a supervised group-based exercise intervention with psychosocial support were more cost-effective than usual care [12]; this study is included in our review. The second review by Mewes et al. [10] also only identified a single study, which concluded that a home-based self-managed exercise intervention was not cost-effective compared to an active control consisting of flexibility and relaxation activities after breast cancer surgery [13]; this study is also included in our review.

Using QALYs as the primary measure of health consequence, the evidence surrounding the cost-effectiveness of exercise and physiotherapy interventions for breast cancer rehabilitation following surgery was equivocal. Three studies reported favourable cost-effectiveness results for different exercise or physiotherapy interventions $[12,28$, 
29]. In contrast, four studies conducted in different patient populations and healthcare settings found that exercise or physiotherapy interventions were not cost-effective using the QALY framework and on the basis of recommended country-specific cost-effectiveness thresholds for the QALY metric [13, 26-28].

Cost-effectiveness evidence was only reported within three countries, each with different healthcare systems (Australia, The Netherlands and France). This cost-effectiveness evidence was largely based on small studies with sample sizes ranging from 60 to 244 women. Methodological variations in recommended approaches across jurisdictions to the conduct of health economic evaluations may partly explain variations in cost-effectiveness results. For example, not all studies using the QALY framework for the analyses estimated QALYs using the same multi-attribute utility measure. The EQ-5D-3L was used in four studies [13, 26-28], whilst one study used utilities derived from the SHE [12] and a further study relied upon an external mapping algorithm [29]. Furthermore, variations in the content and delivery of exercise and physiotherapy interventions and the relative prices of the resource components of those interventions and their resource consequences are also likely to be factors driving the lack of consistency in findings. Consequently, any variation in cost-effectiveness estimates is likely to be driven, at least in part, by variations in methodological factors, as well as variations in the essential features of the interventions evaluated.

The comparators considered by the studies included in this systematic review can broadly be categorised as postoperative exercise versus control $[12,26]$, exercise versus control during adjuvant breast cancer treatment $[13,25$, $27,28]$ and exercise versus control following breast cancer treatment [29]. We found no economic evaluations comparing post-operative early versus delayed exercise interventions despite evidence for their clinical effectiveness [8]. Clearly, there is a need for further research that assesses the cost-effectiveness of the broad range of exercise and physiotherapy interventions that have been developed, many of which are used in routine clinical practice. A particular focus of future research should be to disentangle the contributing effects of frequency, intensity, time and type of exercise and physiotherapy interventions on cost-effectiveness outcomes with the view to specifying the relationship between features of those interventions and cost-effectiveness outcomes. Furthermore, although all but one study included in this systematic review measured health consequences in terms of QALYs, which are widely recommended for cost-effectiveness-based decision-making, there is a need for assessments of the sensitivity of widely used multi-attribute utility measures such as the EQ-5D-3L to changes in outcomes of interest, such as symptoms of fatigue [8].
The key strength of this study is the robust methodology adopted, which included following recommended guidelines for the conduct of systematic reviews of economic evaluations [18], and a transparent approach to study identification, assessment, data extraction and critical appraisal. Variations in methodological approaches and factors precluded the use of meta-analysis for combining cost-effectiveness evidence across studies, in line with other systematic reviews of economic evaluations [35, 36]. The study does have limitations, which should be borne in mind by readers. First, we did not search grey literature databases, including TRIP and Open Grey, within our search strategies. We worked closely with an information specialist to develop, test and refine our search strategies, but cannot preclude the possibility of exclusion of potentially relevant studies. Second, interpretation of the cost-effectiveness assessments that measured health consequences in terms of natural or biomedical units of outcomes, such as changes in BMI or cardiorespiratory fitness [25], is constrained by the absence of external costeffectiveness thresholds for these health consequences. External evidence from stated or revealed preference studies on the value that should be placed on these effects will be required for the purposes of cost-effectiveness-based decision-making.

\section{Conclusion}

This review has highlighted that the evidence base surrounding the cost-effectiveness of exercise and physiotherapy interventions for the treatment of breast cancer remains sparse with contrasting conclusions. Future research should particularly aim to broaden the evidence base by disentangling the contributing effects of frequency, intensity, time and type of exercise and physiotherapy interventions on cost-effectiveness outcomes.

Funding This study was funded by NIHR HTA (RMRCT0127).

\section{Compliance with ethical standards}

Conflict of interest Kamran Ahmad Khan, Bruno Mazuquin, Alastair Canaway, Stavros Petrou and Julie Bruce declare that they have no conflict of interest.

Ethical approval This article does not contain any studies with human participants performed by any of the authors.

Protocol registration PROSPERO registration number CRD42018108978, registered 19/09/2018. 
Open Access This article is distributed under the terms of the Creative Commons Attribution 4.0 International License (http://creativeco mmons.org/licenses/by/4.0/), which permits unrestricted use, distribution, and reproduction in any medium, provided you give appropriate credit to the original author(s) and the source, provide a link to the Creative Commons license, and indicate if changes were made.

\section{References}

1. CRUK Cancer Research UK: Breast Cancer Survival Statistics. http://www.cancerresearchuk.org/cancer-info/cancerstats/types /breast/survival/

2. National Comprehensive Cancer Network (2003) Breast cancer clinical Practice Guidelines in Oncology. JNCCN 1(2):148. https://doi.org/10.6004/jnccn.2003.0016

3. Hortobagyi GN (1998) Treatment of breast cancer. J Med 339(14):974-984. https://doi.org/10.1056/nejm19981001339 1407

4. Lee TS, Kilbreath SL, Refshauge KM, Herbert RD, Beith JMJBCR, Treatment (2008) Prognosis of the upper limb following surgery and radiation for breast cancer. Breast Cancer Res Treat 110(1):19-37. https://doi.org/10.1007/s10549-007-9710-9

5. McNeely ML, Campbell K, Ospina M, Rowe BH, Dabbs K, Klassen TP, Mackey J, Courneya K (2010) Exercise interventions for upper-limb dysfunction due to breast cancer treatment. Cochrane Database Syst Rev 1:1. https://doi.org/10.1002/14651 858.cd005211.pub2

6. Mejdahl MK, Andersen KG, Gartner R, Kroman N, Kehlet H (2013) Persistent pain and sensory disturbances after treatment for breast cancer: six year nationwide follow-up study. BMJ 346:f1865. https://doi.org/10.1136/bmj.f1865

7. Knols R, Aaronson NK, Uebelhart D, Fransen J, Aufdemkampe G (2005) Physical exercise in cancer patients during and after medical treatment: a systematic review of randomized and controlled clinical trials. J Clin Oncol 23(16):3830-3842. https:// doi.org/10.1200/jco.2005.02.148

8. McNeely ML, Campbell KL, Rowe BH, Klassen TP, Mackey JR, Courneya KS (2006) Effects of exercise on breast cancer patients and survivors: a systematic review and meta-analysis. CMAJ 175(1):34-41. https://doi.org/10.1503/cmaj.051073

9. Schmitz KH, Holtzman J, Courneya KS, Mâsse LC, Duval S, Kane R (2005) Controlled physical activity trials in cancer survivors: a systematic review and meta-analysis. Cancer Epidemiol Biomark Prev 14(7):1588

10. Mewes J, Steuten LMG, Ijzerman MJ, van Harten WH (2012) Effectiveness of multidimensional cancer survivor rehabilitation and cost-effectiveness of cancer rehabilitation in general: a systematic review. Oncologist 17:1-14

11. Roine E, Roine RP, Räsänen P, Vuori I, Sintonen H, Saarto T (2009) Cost-effectiveness of interventions based on physical exercise in the treatment of various diseases: a systematic literature review. Int J Technol Assess Health Care 25(4):427-454. https://doi.org/10.1017/S0266462309990353

12. Gordon LG, Scuffham P, Battistutta D, Graves N, Tweeddale M, Newman B (2005) A cost-effectiveness analysis of two rehabilitation support services for women with breast cancer. Breast Cancer Res Treat 94(2):123-133

13. Haines TP, Sinnamon P, Wetzig NG, Lehman M, Walpole E, Pratt T, Smith A (2010) Multimodal exercise improves quality of life of women being treated for breast cancer, but at what cost? Randomized trial with economic evaluation. Breast Cancer Res Treat 124(1):163-175. https://doi.org/10.1007/s1054 9-010-1126-2
14. Assessment CCOfHT (2006) Guidelines for economic evaluation of pharmaceuticals: Canada, 3rd edition. Canadian Coordinating Office for Health Technology Assessment

15. Consortium SM (2013) Guidance to manufacturers for completion of New Product Assessment Form (NPAF), 2013

16. Pharmaceutical Benefits Advisory Committee \%J Canberra APBAC (2002) Guidelines for the pharmaceutical industry on preparation of submissions to the Pharmaceutical Benefits Advisory Committee

17. NICE U (2013) Guide to the methods of technology appraisal. National Institute for Health and Clinical Excellence (NICE) London, UK

18. Moher D, Liberati A, Tetzlaff J, Altman DG (2009) Preferred reporting items for systematic reviews and meta-analyses: the PRISMA statement. Ann Internal Med 151(4):264-269

19. Husereau D, Drummond M, Petrou S, Carswell C, Moher D, Greenberg D, Augustovski F, Briggs AH, Mauskopf J, Loder E, Force obotCT (2013) Consolidated health economic evaluation reporting standards (CHEERS) statement. Cost Eff Resour Alloc 11(1):6. https://doi.org/10.1186/1478-7547-11-6

20. Higgins JP, Altman DG, Gøtzsche PC, Jüni P, Moher D, Oxman AD, Savović J, Schulz KF, Weeks L, Sterne JAJB (2011) The Cochrane Collaboration's tool for assessing risk of bias in randomised trials. BMJ 343:d5928

21. Husereau D, Drummond M, Petrou S, Carswell C, Moher D, Greenberg D, Augustovski F, Briggs AH, Mauskopf J, Loder EJVIH (2013) Consolidated health economic evaluation reporting standards (CHEERS) - explanation and elaboration: a report of the ISPOR health economic evaluation publication guidelines good reporting practices task force. Value Health 16(2):231-250

22. Stout NL, Pfalzer LA, Springer B, Levy E, McGarvey CL, Danoff JV, Gerber LH, Soballe PW (2012) Breast cancerrelated lymphedema: comparing direct costs of a prospective surveillance model and a traditional model of care. Phys Ther 92(1):152-163. https://doi.org/10.2522/ptj.20100167

23. Su CC, Remedios C (2015) Clinical and cost effectiveness of additional post-operative physiotherapy on enhancing shoulder range and functional arm use in breast cancer patients. Physiotherapy 1:71-72

24. Kampshoff CS, van Dongen JM, van Mechelen W, Schep G, Vreugdenhil A, Twisk JWR, Bosmans JE, Brug J, Chinapaw MJM, Buffart LM (2018) Long-term effectiveness and costeffectiveness of high versus low-to-moderate intensity resistance and endurance exercise interventions among cancer survivors. J Cancer Survivorship 12(3):417-429. https://doi.org/10.1007/ s11764-018-0681-0

25. Perrier L, Foucaut A, Touillaud M, Kempf-Lepine AS, Morelle M, Heinz D, Gomez F, Meyrand R, Baudinet C, Berthouze S, Carretier J, Denizot-Guillemaut S, Perol D, Tredan O, Philip T, Reynes E, Bachmann P, Fervers B (2016) A cost-effectiveness analysis of a 6-month physical activity program versus usual dietary care during adjuvant chemotherapy in breast cancer patients. Value Health 19(3):A149

26. Gordon LG, DiSipio T, Battistutta D, Yates P, Bashford J, Pyke C, Eakin E, Hayes SC (2017) Cost-effectiveness of a pragmatic exercise intervention for women with breast cancer: results from a randomized controlled trial. Psychooncology 26(5):649-655. https://doi.org/10.1002/pon.4201

27. May AM, Bosch MJ, Velthuis MJ, van der Wall E, Steins Bisschop CN, Los M, Erdkamp F, Bloemendal HJ, de Roos MA, Verhaar M, Ten Bokkel Huinink D, Peeters PH, de Wit GA (2017) Cost-effectiveness analysis of an 18-week exercise programme for patients with breast and colon cancer undergoing adjuvant chemotherapy: the randomised PACT study. BMJ Open 7(3):e012187. https://doi.org/10.1136/bmjopen-2016-012187 
28. van Waart H, van Dongen JM, van Harten WH, Stuiver MM, Huijsmans R, Hellendoorn-van Vreeswijk JAJH, Sonke GS, Aaronson NK (2018) Cost-utility and cost-effectiveness of physical exercise during adjuvant chemotherapy. Eur $\mathbf{J}$ Health Econ 19(6):893-904. https://doi.org/10.1007/s1019 8-017-0936-0

29. Mewes JC, Steuten LMG, Duijts SFA, Oldenburg HSS, Van Beurden M, Stuiver MM, Hunter MS, Kieffer JM, Van Harten WH, Aaronson NK (2013) Cost-effectiveness of cognitive-behavioral therapy and physical exercise for alleviating treatment-induced menopausal symptoms in breast cancer patients. Value Health 16(3):A141

30. Briggs A, Sculpher M, Claxton K (2006) Decision modelling for health economic evaluation. Oxford University Press, Oxford

31. Drummond MF, Sculpher MJ, Claxton K, Stoddart GL, Torrance GW (2015) Methods for the economic evaluation of health care programmes. Oxford University Press, Oxford

32. Duijts SF, van Beurden M, Oldenburg HS, Hunter MS, Kieffer JM, Stuiver MM, Gerritsma MA, Menke-Pluymers MB, Plaisier PW, Rijna HJJOCO (2012) Efficacy of cognitive behavioral therapy and physical exercise in alleviating treatment-induced menopausal symptoms in patients with breast cancer: results of a randomized, controlled, multicenter trial. J Clin Oncol 30(33):4124-4133

33. Hürny C, van Wegberg B, Bacchi M, Bernhard J, Thürlimann B, Real O, Perey L, Bonnefoi H, Coates A (1998) Subjective health estimations (SHE) in patients with advanced breast cancer: an adapted utility concept for clinical trials. Br J Cancer 77:985. https ://doi.org/10.1038/bjc.1998.162

34. Husereau D, Drummond M, Petrou S, Carswell C, Moher D, Greenberg D, Augustovski F, Briggs AH, Mauskopf J, Loder E (2013) Consolidated health economic evaluation reporting standards (CHEERS) statement. BMJ 346:1. https://doi.org/10.1136/ bmj.f1049

35. Gilbody S, Bower P, Whitty P (2006) Costs and consequences of enhanced primary care for depression: systematic review of randomised economic evaluations. Br J Psychiatry 189(4):297-308. https://doi.org/10.1192/bjp.bp.105.016006

36. Drummond MF, Iglesias CP, Cooper NJ (2008) Systematic reviews and economic evaluations conducted for the National Institute for Health and Clinical Excellence in the United Kingdom: a game of two halves? Int J Technol Assess Health Care 24(2):146-150. https://doi.org/10.1017/S0266462308080203

Publisher's Note Springer Nature remains neutral with regard to jurisdictional claims in published maps and institutional affiliations. 